\title{
Axel HONNETH, Le Droit de la liberté. Esquisse d'une éthicité démocratique
}

Aude Malkoun-Henrion

\section{(2) OpenEdition}

\section{Journals}

Édition électronique

URL : http://journals.openedition.org/ress/3579

DOI : $10.4000 /$ ress.3579

ISBN : $1663-4446$

ISSN : $1663-4446$

Éditeur

Librairie Droz

Édition imprimée

Date de publication : 30 novembre 2016

Pagination : 316-318

ISSN : 0048-8046

Référence électronique

Aude Malkoun-Henrion, "Axel HONNETH, Le Droit de la liberté. Esquisse d'une éthicité démocratique », Revue européenne des sciences sociales [En ligne], 54-2 | 2016, mis en ligne le 05 septembre 2016, consulté le 23 septembre 2020. URL : http://journals.openedition.org/ress/3579 ; DOI : https://doi.org/ 10.4000/ress.3579

Ce document a été généré automatiquement le 23 septembre 2020.

(C) Librairie Droz 


\title{
Axel HONNETH, Le Droit de la liberté. Esquisse d'une éthicité démocratique
}

\author{
Aude Malkoun-Henrion
}

\section{RÉFÉRENCE}

Axel HONNETH, 2015, Le Droit de la liberté. Esquisse d'une éthicité démocratique, Paris, Gallimard, « NRF Essai », 608 p.

1 Le Droit de la liberté, paru en 2011 en allemand, marque, près de 20 ans après La Lutte pour la reconnaissance un moment-clé dans la pensée d'Axel Honneth. Ce dernier ouvrage de 1992 déployait la notion hégélienne de reconnaissance. S'appuyant sur la psychologie sociale de Mead et la théorie de la relation d'objet de Winnicott, il y distinguait, à la suite du jeune Hegel, trois sphères d'interactions sociales au sein desquelles se dégagent trois modèles de reconnaissance mutuelle (amour, droit, solidarité), toutes nécessaires, à différents niveaux, à l'acquisition de l'autonomie et au développement d'un rapport pratique positif à soi (confiance en soi, respect de soi, estime de soi). Ceci le menait à poser les dénis de reconnaissance comme des pathologies, notamment des expériences concrètes de mépris, constituant, en grande partie, le cœur des conflits sociaux de la modernité. Dans la lignée de ce modèle du conflit social, Honneth cherche à élaborer dans Le Droit de la liberté une notion de la justice adaptée à un tel modèle de lutte pour la reconnaissance. Cette nouvelle œuvre opère, dans la construction de sa théorie de reconnaissance, deux déplacements. D'abord, la notion de reconnaissance semble subsumée sous celle de liberté: les différents modèles de reconnaissance sont intégrés à ce qu'il nomme la «liberté sociale ». Ensuite, Honneth accorde une plus grande importance aux institutions modernes et à leur histoire.

2 Cette évolution n'est pas sans rapport à l'ancrage hégélien de sa théorie qu'il consolide ici. En effet, Honneth revient à Hegel, notamment à sa Philosophie du droit, duquel il reprend deux thèses majeures: celle de la coexistence, au sein de la modernité, de 
divers sens de la liberté et celle du rôle déterminant des institutions dans l'effectivité, pour les individus, de cette dernière. L'autre emprunt à Hegel est de type méthodologique. Ici, Honneth insiste sur la nécessité, pour la philosophie sociale, d'adopter une démarche hégélienne dans l'élaboration d'une théorie de la justice. Démarche qu'il nomme «reconstruction normative » (p. 22) : une théorie de la justice n'a pas pour tâche de prescrire au monde des normes tirées de principes abstraits mais bien plutôt d'identifier et de critiquer celles à l'œuvre dans la réalité sociale et produites par les conflits qui la traversent. Par l'analyse immanente de «tout un tissu de pratiques sociales » (p. 19), une telle théorie doit reconstruire, et non construire, ces valeurs et idéaux "institutionnalisés » (p.18) sur lesquels repose toute société. Du même coup, la philosophie sociale retrouvera sa puissance "critique»: elle pourra révéler les décalages entre ces principes et leur réalisation, généralement déformée.

La structure du livre est rigoureuse et se divise en trois parties imbriquées conceptuellement. La première («Une revisitation historique : le droit de la liberté ») développe la notion de liberté (autonomie individuelle), concept central des théories modernes de la justice. Honneth y dégage, historiquement, deux interprétations de la liberté ayant donné lieu à deux conceptions de la justice dominantes. La liberté négative (Hobbes, Locke, Nozick), ou absence de contrainte extérieure, renvoie à une conception atomiste du sujet et définit la justice comme l'organisation de ces atomes. La liberté réflexive (Rousseau, Kant), ou autodétermination, comprend la justice comme un ensemble de procédures permettant la coopération des individus. Honneth rappelle ici la critique hégélienne de ces deux libertés. Concernant la liberté négative, il souligne que non seulement cette dernière "s'arrête au seuil véritable de l'auto-détermination individuelle ", mais qu'elle ne " rend pas [non plus l'individu] capable de se poser à soimême les objectifs qu'il s'agira pour lui de réaliser dans le monde » (p. 51). Quant aux idées de liberté réflexive, Honneth montre qu'elles « n'ont pas prise sur les conditions permettant en premier lieu [leur] exercice » puisqu'aucun modèle de liberté réflexive "n'interprète comme des composantes de la liberté elle-même les conditions sociales " qui la rendent possible (p.69). Honneth leur oppose une troisième forme, liée à la notion de reconnaissance mutuelle, la liberté «sociale» (Hegel, Marx) ou « intersubjective»: les sujets sont effectivement libres lorsqu'ils sont en mesure de former et réaliser leurs projets et désirs au sein de rapports institués de reconnaissance. Ainsi, cette liberté est « sociale » dans la mesure où « elle envisage une institution bien déterminée de la réalité sociale non plus comme un pur et simple additif à la liberté, mais comme le médium et la condition de la mise en application de cette liberté. » (p. 71).

4 En deuxième partie («La possibilité de la liberté »), Honneth, afin de dégager les conditions de possibilité d'une liberté effective, montre les limites des deux premières formes de liberté. Celles-ci, légitimes mais abstraites, entraînent, lorsqu'elles sont hypostasiées, diverses pathologies de la liberté (juridisme, moralisme) qui correspondent à la négation du caractère intersubjectif et multiforme de cette dernière.

Dans la troisième et dernière partie ("La réalité de la liberté »), Honneth procède à la reconstruction du mouvement concret de la liberté au sein des trois sphères de reconnaissance. Il dresse à cette occasion, à l'aide des modèles de Weber et de Parsons ainsi que de références fictionnelles (littérature et cinéma), un saisissant tableau de la modernité. Une modernité constituée de "luttes menées au nom de la réalisation 
sociale des promesses de liberté intrinsèques » (p. 504) aux sphères sociales, et contre les structures traditionnelles de la société mais aussi contre le juridisme et le moralisme ambiants. Ce tableau lui permet de poser les conditions de réalisation et de reproduction d'une société qui ne soit pas simplement l'articulation d'atomes égoïstes.

6 Ce livre, particulièrement brillant, offre une solide illustration de la puissance explicative et critique du modèle de la reconnaissance mutuelle. Il présente également une éclatante démonstration de la pluralité des significations de la liberté et de la nécessité de tenir compte de chacune, notamment dans l'élaboration d'un concept de justice sociale qui ne soit pas coupé des institutions sociales ni des conflits recognitifs concrets. Cette idée que la liberté politique est déterminée par d'autres formes (morales, juridiques, sociales) de liberté - idée que Honneth ne cesse de déployer depuis La Lutte pour la reconnaissance -, constitue ainsi la force majeure de l'ouvrage. Tout au plus, déplorera-t-on qu'il ne soit pas fait plus de place à la critique - toujours pertinente - de Nancy Fraser, selon laquelle une véritable reconnaissance politique n'est possible que si elle passe également, au niveau socio-économique, par une redistribution du capital.

7 Aussi, s'il est possible de s'interroger sur le caractère "occidentalo-centrique " (reconnu) de la notion d'éthicité démocratique développée par Honneth, ou de regretter la faiblesse de sa critique à l'endroit de la financiarisation généralisée du capitalisme contemporain, il n'en demeure pas moins que Le Droit de la liberté s'annonce comme un classique de la philosophie sociale.

\section{AUTEURS}

\section{AUDE MALKOUN-HENRION}

Université de Paris 1-Sorbonne, NOSOPHI - Université de Montréal, CRE 